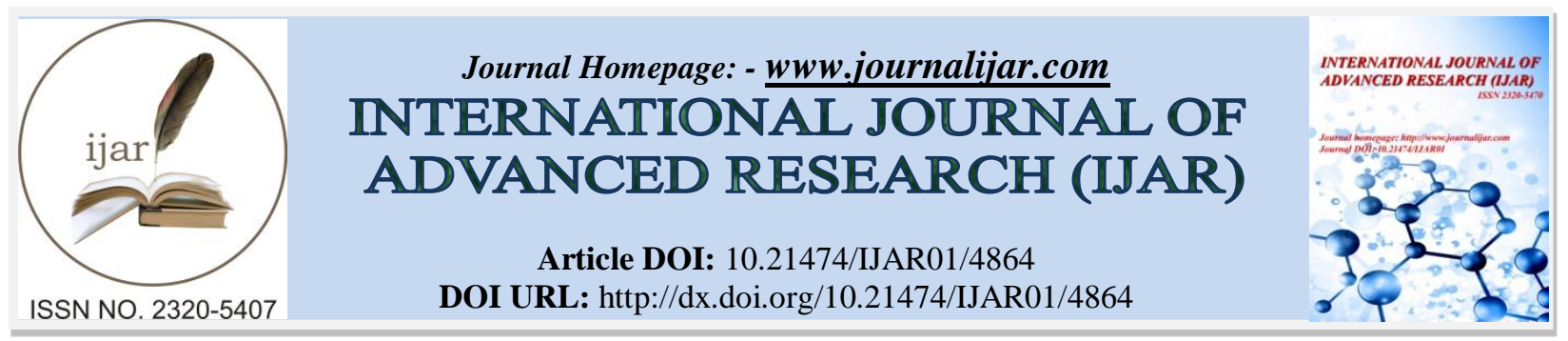

RESEARCH ARTICLE

\title{
KNOWLEDGE, ATTITUTE AND PRACTICES ON SCABIES AMONG RELIGIOUS SCSHOOL (MADRASA) HOSTEL STUDENTS OF HELMAND PROVINCE AFGHANISTAN.
}

Dr. Ubaidullah Dawoodzada.

Jodhpur School of Public Health (JSPH) Maulana Azad University Jodhpur, India.

\section{Manuscript Info}

\section{Manuscript History}

Received: 16 May 2017

Final Accepted: 18 June 2017

Published: July 2017

\section{Abstract}

Rational: Unhygienic living condition, poor sanitation, over crowed rooms, low level of knowledge of disease prevention, and poor sanitation puts more than 1300 madrasa students at risk of Scabies who are living as group in a dormitory. Student from Helmand and neighboring provinces are enrolled in this madrasa and are staying in dormitory for more than one year. The point prevalence rate of scabies in this school is $15 \%$. This issues need attention/follow up and have great public health importance and to plan for improvement.

Objective: The objective of this study was to explore the knowledge, attitudes and practices about scabies in religious school (Madrasa) of Helmand province Afghanistan.

Method: A descriptive cross-sectional study was performed. Specifically designed KAP questionnaire used. Consent letter signed with participants. Questionnaire was translated into local language. Ms. Excel used for data entry/analysis. Double entry of data completed. $10 \%$ of data cross checked for accuracy. The surveyors were trained on interview, data collection methods. 433 students randomly selected (33.3\% of total).

Results: More than 80\% participants aged 10-20 years. $44 \%$ reported that they are informed about scabies, 56\% do not know about scabies. $62.9 \%$ got the knowledge through mass media and $37.1 \%$ reported are informed through medical professional, family and friends. Scabies is serious (16\%) not serious (24\%) did not know about the seriousness (39\%). Diseases spread through sharing cloth and blankets (44\%).

Conclusions: Low level of knowledge about the diseases (44\%), public health workers should give priority to health education, media play good role in dissemination of information. Proper communication on scabies prevention through Radio (available in school and villages). Health workers to develop good network for support with the madrasa and communities in similar situations. People with scabies should be encouraged to seek medical help and avoid sharing cloths and blankets.

Copy Right, IJAR, 2017,. All rights reserved. 


\section{Acknowledgements:-}

I, the author of this paper would like to express my sincere gratitude to my professors at Jodhpur School of Public Health (JSPH), Maulana Azad University, Dr Latika Nath Sinha, Dr Nitin Joshi, Dr Pramila Vivek, who taught me how to conduct a research and provided me with valuable comments on my work. Besides I gratefully acknowledge the contribution of Dr Rahmatullah Mohammadi my co-guide for his very systematic input in finalization of this study.

I also would like to thank the team (Data collectors and Data entry officers) who participated in this work. I also would like to thank the Mr. Safiullah in charges of Madrasa (The religious school of Helmand) and his team for being available to participate in the study. Finally, I want to acknowledge all the selected students who were interested to provide best and accurate responses.

I hope this research could provide basic information on the control of scabies especially for this public health officer who are supporting vulnerable group who live in hostels and prisoners. I hope to contribute in the enhancement of quality of health service delivery and control of scabies.

\section{Background and Rationale:-}

More than 1300 students are in risk to be affected by skin disease of scabies due to poor hygiene, sanitation, poor living style, poor knowledge of personal and environment hygiene and cleanness. The school is established in year 2002 aiming to promote religious values and trained children on Islamic principle of worship, Islamic social values and the Islamic rules and regulation for public justice among the Muslim community. Students from Helmand, Kandahar, Zabul, Nimroz and Urozgan provinces are enrolled in this madrasa. As there are Students who are coming from neighboring provinces they have to stay overnight in the madrasa for the period of their education ( 2-4 years). As the madras is open for all population and all families who are willing to send their children for religious education they are accepting student as much as referred to this madrasa and those who are approaching this madrasa for training.

The principal of the madrasa do not have a plan for total number of Students to be trained or they do not have the ceiling for maximum number of the students. Therefore, student's accommodation and feeding become a challenge. The school does not have enough resources to allocate separate beds and blanket to each student and most of the time they are sharing beds and blankets.

Blankets, quilts and mattresses are used several times with no plan and action for cleaning the bed cover and poor plan for washing the blankets. Due to low level of knowledge of the students and their facilitators on hygiene and sanitation essential steps for keeping the hygiene is not followed. The chance of infection by scabies parasites spreading among the students and even teachers are affected. During the cross-sectional survey conducted by public health department of Helmand in the month of May 2016, it was determined that the point prevalence rate of scabies in the mentioned school is $15 \%$. The total number of student in May was 1300 out of which 200 of them were suffering from scabies. The school does not have a fixed budget and resources obtained from the Ministry of Education are not covering the minimum requirement for healthy lives in this environment. The school also received donation in form of cash and in-kind from the public for their foods and other needs. As the education level of school managers and facilitators on the issues of managing a group and leading a training centers is very much poor and they could not make the best use of the available resources. Despite that the weather of Helmand provinces is suitable for hygiene maintenance and it is possible to bath almost every day and to wash the cloths, blankets and mattresses more often and more frequent but they are not practicing these hygienic values.

Scabies is a contagious skin infection that spreads rapidly and worldwide poor communities are affected by scabies.

Personal hygiene is an important preventive measure and access to adequate water supply is important in control of Scabies. Epidemics have been linked to poverty, poor water-supply, and sanitation and overcrowding. Scabies cases reported from all provinces in Afghanistan. Unfortunately report on the number of Scabies cases is not systematic and the Scabies is reporting in the HMIS (Health Management information System) under itching and is covering a wide range of skin diseases. 
Technical guideline for scabies prevention and treatment is included in the training of mid-level health workers, nurses, midwives, assistant doctors, doctors and community health workers. Training on scabies case management and prevention is provided in all training institutions (pre-services and in-serves training).

\section{Literature Review:-}

WHO estimates that the disease has impacted on 7,000 residents in Nusai district in Badakhshan province," Dr Sardar Ahmad, WHO information officer reported in June 2016. WHO and the Afghan Ministry of Public Health $(\mathrm{MOPH})$ were sending medication and medical team to the area to stop the spread of the disease. Scabies is a fairly common infectious disease of the skin caused by a mite, producing pimple-like irritations. Scabies mites are transferred by direct skin-to-skin contact. The most prominent symptom of scabies is intense itching, particularly at night. The areas of the skin most affected include the webs and sides of the fingers, around the wrists, elbows and armpits, waist, thighs, genitalia, breasts and the lower buttocks.

WHO, in collaboration with other partners and the Ministry of Health, has taken the initiative to combat the crisis by sending medicines and supplies to the affected area, Sardar Ahmad said, noting that they were starting health education awareness programe to prevent a re-occurrence of the disease in such remote areas.

Summary/conclusion of an article published in Journal of clinical case studies of Anglo State University, USA (Author Sangeeta Singg) reported on the Scabies as follows:

The present survey confirmed that most participants (94\%) were unaware about the facts about scabies, perhaps due to the neglected status of the disease. A scabies awareness and educational program might be of special importance for the very poor who are disproportionately affected and other vulnerable groups such as children, elderly, sick and homeless persons. The data supported the assumption that women are more afraid of contracting and living with scabies than men, perhaps due to cultural issues. Thus, women would benefit from psychosocial support.

Scientific community and health care providers need to assess systematically not only the physiological burden, but also the psychosocial burden of scabies and devise a multifaceted intervention program.

\section{Objectives:-}

The objective of this study is to explore the knowledge, attitudes and practices about Scabies Disease in religious school (Madrasa) hostel students of Helmand province, Afghanistan.

\section{Study Design:-}

This is a cross-sectional study which is conducted in school (Madrasa) student's hostel in Helmand province of Afghanistan in order to explore the knowledge, attitudes and practices about scabies.

\section{Target Respondents/participant;-}

The research population or the sampling frame was targeted students in religious school that were 433 persons.

\section{Sample size:-}

Random sampling method (every third student) selected for interview in this study, (433 students selected from 1300 students.

\section{Duration of the study:-}

This study took a period of 32 weeks.

\section{Research Methodology:-}

A cross section study is conducted to determine the knowledge, attitude and practices of religious students in Helmand Province of Afghanistan. The study targeted every third students from general registers of Religious school of Helmand Province, Consent letter signed with all and data obtained. The Ms. Excel was used for data entry and data analysis. Double entry of data completed and $10 \%$ of the questionnaires were cross checked for more validation and accuracy. To present a better picture objective of the study we planned to collect data from random sampling of religious students, 433 participants were all male. 


\section{Study Tools:-}

The KAP Questionnaire designed by WHO was adapted to Helmand situation and was applied in the study. All questions were covering four sections dealing with the socio-demographic situation of respondents and the following aspects related to Scabies disease, knowledge, behavior toward the possibility of contracting of scabies, and attitudes toward patients with scabies.

\section{Pilot Testing:-}

Data collection instrument/tool, the KAP questionnaire, is developed in English. The tool is then translated in to local languages (Pashtu) and back it is retranslated to English to ensure the quality of translation. A pre-test of the study tools is considered in order to check the tools (questionnaires) and to identify and solve the unforeseen problems before the actual data collection. The pre-test emphasized to improve the translation, check accuracy and adequacy of the questionnaire, and to know the time of the interview for each questionnaire. In addition, Interviewer pre-test at the training room is also ensured.

\section{Training:-}

Two days training is conducted for field researchers (4 teachers) who were the staff of religious school in Helmand Province, The training emphasized on the data collection tools, survey method, and piloting. The training is conducted in one batch. Necessary training material is provided to the participants during the training. Different methodologies are used in order to make sure the participants understand the objective of the training such as presentation, flip chart and role play. The training is provided in one local language (Pashtu). In order to ensure the knowledge of participants on the survey tools a reliability testing training is also taken from all participants.

\section{Data collection:-}

Four Field researchers (teachers) collected data from 433 religious students out of 1300. To minimize the potential for social desirability bias, the interviewer explained the purpose, confidentiality and anonymity of the study to each provider before seeking consent, after a consent form is signed by each participant, data collection took a period of 4weeks simultaneously by all interviewers and finally after data collection is completed, an excel data base is prepared where all the data from the questionnaires are entered into and then accordingly analyzed.

\section{Monitoring and supervision:-}

The process of data collection are monitored and supervised by me and principle investigator during data collection period and at least $10 \%$ of the data are rechecked by principle investigator \& me as supervisor.

\section{Date Analysis:-}

I used double data entry mechanism for each filled questionnaire to minimize keypunch errors and ensured the quality of data entered into excel made software. For analyzing purpose all data from the questionnaires are entered into excel base software at the main office. Data are edited and cleaned by principle investigator, and then the data are transferred for further analysis purpose.

\section{Ethical Consideration:-}

This study cannot be the representative of all Afghanistan therefore it did not require MOPH Institutional Review Board (IRB). However, for ethical purpose all data are stored in a safe place and only authorized staff has access to the data bank. The individual information of respondent is kept confidential and not disclosed during the analysis or dissemination phases. Informed consent note in written were taken from each respondent during the interview and data collection. The respondents are informed about their rights during the study.

\section{Results and Key findings:-}

The results of this survey are presented into three main areas as following:

1. Socio-demographic characteristics of respondents

2. knowledge and awareness of respondents

3. Behaviors and attitude of respondents

\section{Socio-demographic characteristics:-}

A total of 433 religious School hostel students were interviewed in Helmand Province, we were able to obtain $33.3 \%$ (every third students randomly selected from 1300) response rate from all the students. Table 1. Below 
describe the Socio-demographic characteristics of respondents as following which were different in terms of awareness level of education.

\begin{tabular}{|c|c|c|}
\hline Categories of information & $\mathrm{N}$ & $\%$ \\
\hline \multicolumn{3}{|c|}{ Which province are you from? } \\
\hline a. Helmand & 301 & 70 \\
\hline b. Kandahar & 92 & 21 \\
\hline c. Urozgan & 29 & 7 \\
\hline d. Ghor & 11 & 3 \\
\hline \multicolumn{3}{|l|}{ Age in Years: } \\
\hline $10-15$ & 154 & 35.6 \\
\hline b. $\quad 15-20$ & 207 & 47.8 \\
\hline $20-25$ & 72 & 16.6 \\
\hline $25-30$ & 0 & 0.0 \\
\hline \multicolumn{3}{|l|}{ How far do you live from the } \\
\hline \multicolumn{3}{|c|}{ nearest health clinic or hospital : } \\
\hline 0-10 kilometers & 433 & 100 \\
\hline 11-20 kilometers & 0 & 0 \\
\hline $21-30$ kilometer & 0 & 0 \\
\hline More than 30 kilometers & 0 & 0 \\
\hline
\end{tabular}

Knowledge and awareness of respondents:-

From the total population interviewed $44 \%$ reported that they are informed about the disease while $56 \%$ reported that they do not know about the disease, meanwhile $16 \%$ stated that the disease is very serious, $21 \%$ stated that it is somewhat serious and the rest $24 \%$ reported that it is not very serrious, $39 \%$ reported do not know,

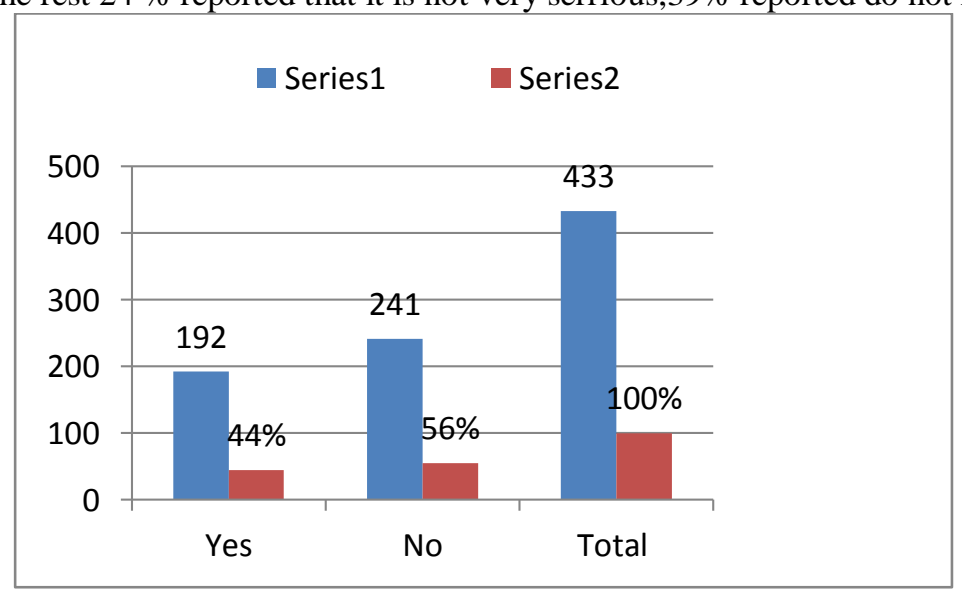

Scabies knowledge and awareness

\begin{tabular}{|l|l|l|}
\hline Description & N & \% \\
\hline Yes & 192 & 44 \\
\hline No & 241 & 56 \\
\hline Total & $\mathbf{4 3 3}$ & $\mathbf{1 0 0}$ \\
\hline
\end{tabular}

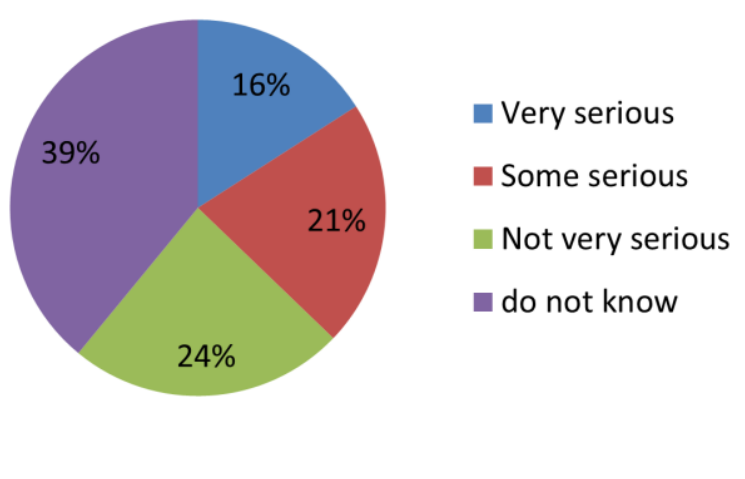


Furthermore Table 3 below described the information about the health seeking behavior of the population under the study:

Table 3:-Health seeking behavior

\begin{tabular}{|c|c|c|}
\hline Health- seeking behavior & $\mathrm{N}$ & $\%$ \\
\hline Go to health facility & 292 & $67 \%$ \\
\hline Go to pharmacy & 48 & $11 \%$ \\
\hline Go to traditional healer & 81 & $19 \%$ \\
\hline Pursue other self-treatment options & 0 & $0 \%$ \\
\hline Other & 12 & $3 \%$ \\
\hline Total & 433 & $100 \%$ \\
\hline
\end{tabular}

About the symptoms of scabies; Chart.3 below describes the number of study population who reported the symptoms as following:

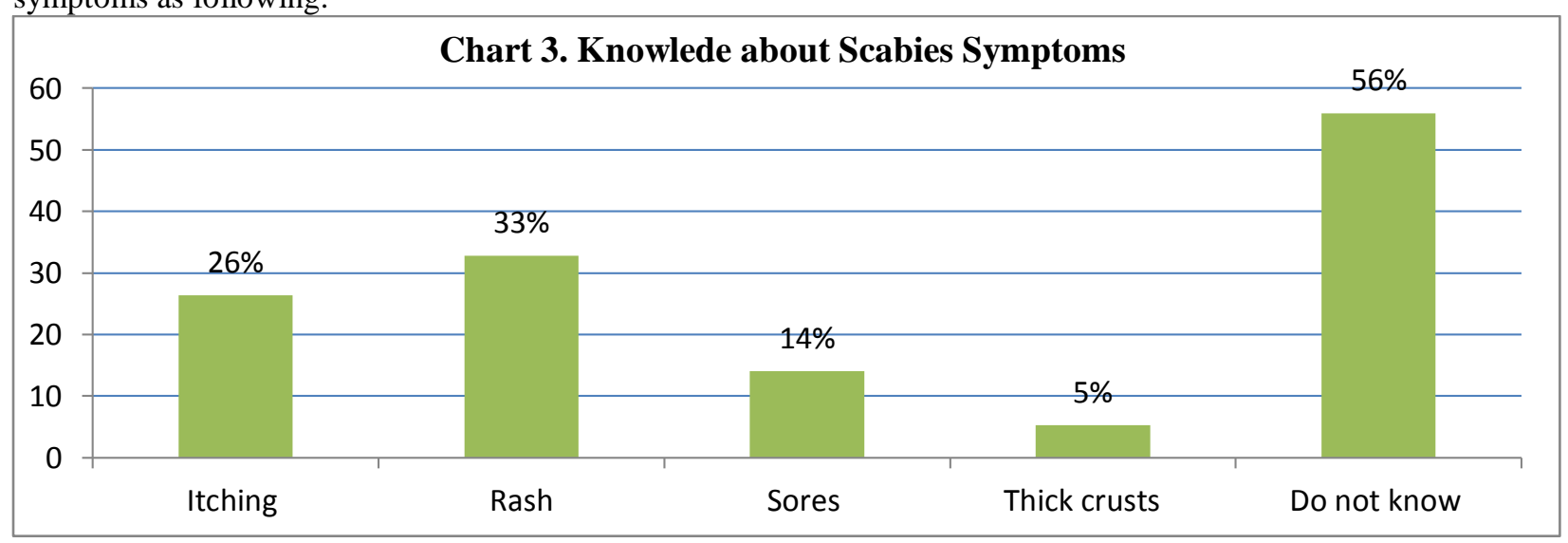

Chart \# 3 about knowledge of scabies symptoms shows that 26\% reported itching, 33\% rash, 14\% sores, 5\% thick crusts and $56 \%$ of respondents were do not know of the symptoms of scabies.

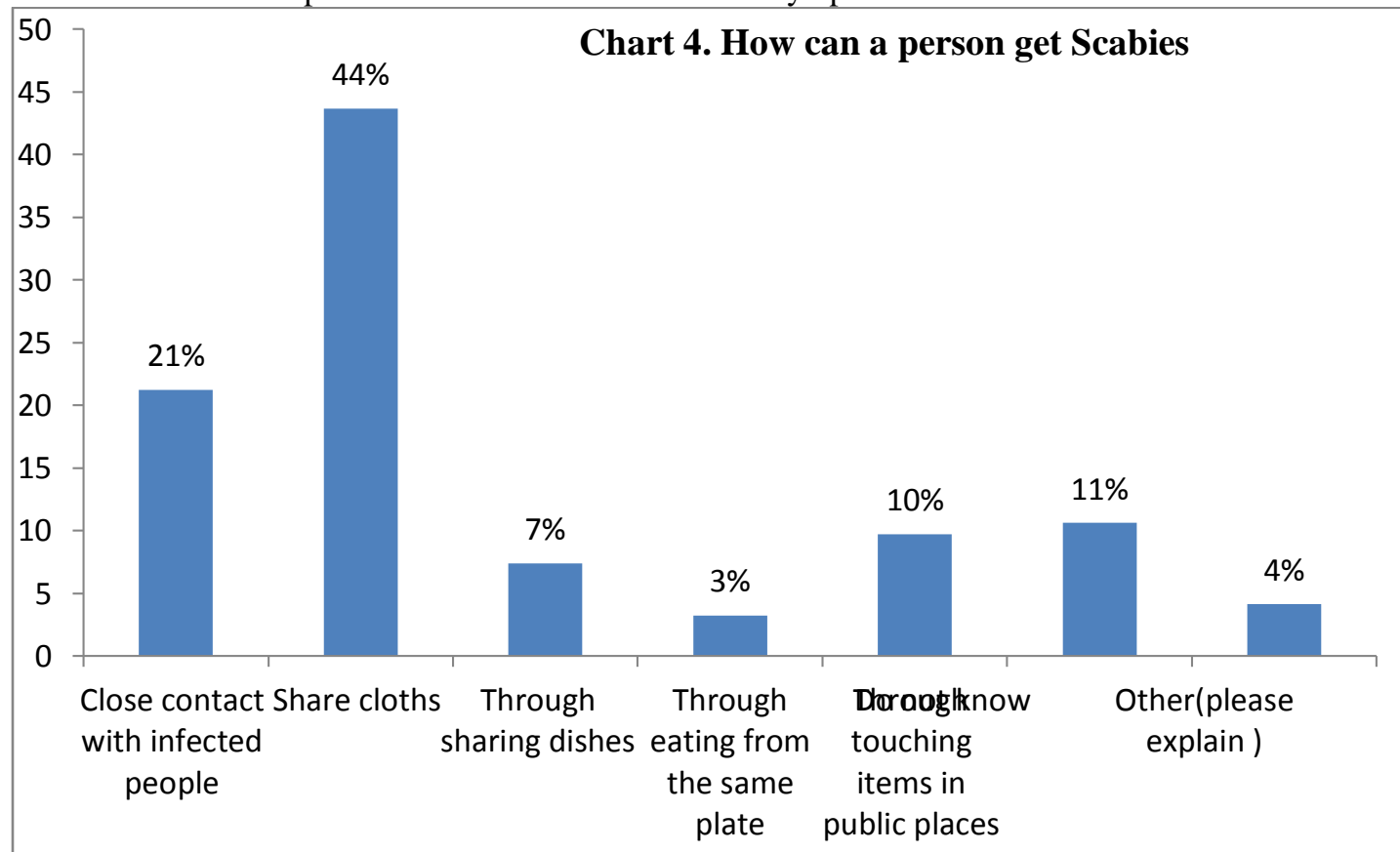


In response to a question that how can a person get scabies, $21 \%$ reported that close contact with infected people , $44 \%$ mentioned by share cloths, $7 \%$ were of the opinion that scabies spread through sharing dishes, $3 \%$ through eating from the same plate.

Table 4:- How can a person Prevent getting scabies (checked all that are mentioned)

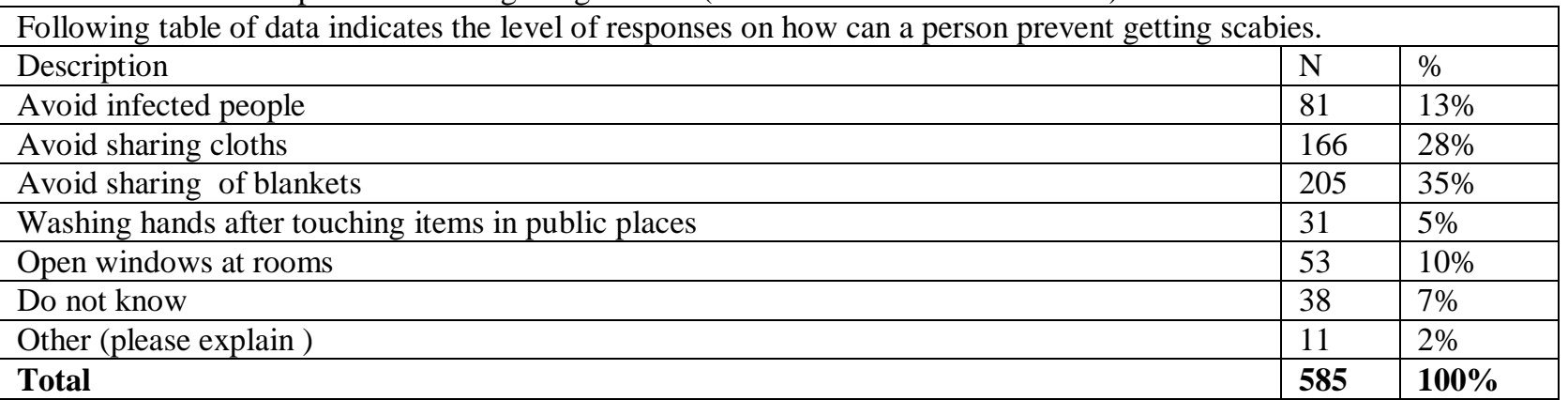

NOTE: total number is 585 in this table and this is because that each respondent were answering more than one question.

The participants response of the ways and means of scabies prevention shows that $35 \%$ reported to avoid sharing of blankets, $28 \%$ mentioned to avoid sharing cloths, $13 \%$ thinks to avoid infected people, $10 \%$ reported to keep widows open for better ventilation, $5 \%$ reported washing hands after touching items in public places, $7 \%$ reported that they do not know and other $2 \%$ (sleeping, kissing, hand shaking....)

The response on the question of how someone with Scabies be cured? Indicates that $56 \%$ of them know that scabies will be cured and $44 \%$ thinks it is not curable

\begin{tabular}{|l|l|l|}
\hline \multicolumn{2}{|l|}{ Table 5. can scabies cured } \\
\hline Description & N & $\%$ \\
\hline Yes & 243 & 56 \\
\hline No & 190 & 44 \\
\hline Total & $\mathbf{4 3 3}$ & $\mathbf{1 0 0 \%}$ \\
\hline
\end{tabular}

\section{Behavior and attitude of Respondents:-}

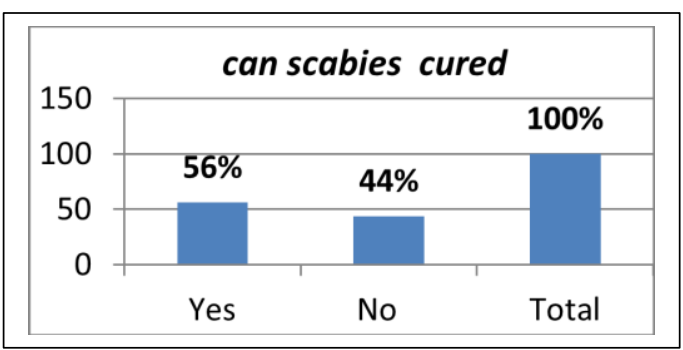

The results about the behaviors and attitude of respondents if they got Scabies indicates that (27\%) would talk about their disease with the parents, $24 \%$ will seek help from doctors/health workers, $22 \%$ will go to a teacher, $13 \%$ will consult their close friends.

Where to go for scabies treatment?

Sixty-seven percent will go to health facility, $19 \%$ will go to traditional healer, $11 \%$ will go to pharmacies, and $3 \%$ will go to shrine and religious leaders.

How they feel and react toward the patients of Scabies?

Twenty seven percent feel compassion and desire to help, $48 \%$ feel compassion but will stay away from these patients, $40 \%$ do not care, $14 \%$ fear from the Scabies patient, $6 \%$ said I have no particular feeling.

In response to the question that what will be the community reaction against person who have scabies? $11 \%$ reported that most people reject him or her, 74\% mentioned that most people are friendly, but they generally try to avoid him or her, $15 \%$ mentioned the community mostly supports and helps him or her.

\begin{tabular}{|l|l|l|}
\hline Below table of Behaviours and attitude of respondents & No & \% \\
\hline 14. Who would you talk to about your illness if you had scabies? & 104 & 24 \\
\hline Doctor or other medical worker & 118 & 27 \\
\hline Parent & 97 & 22 \\
\hline Teacher & 58 & 13 \\
\hline Other family member & & 57 \\
\hline
\end{tabular}




\begin{tabular}{|l|l|l|}
\hline Close friend & 56 & 13 \\
\hline No one & 0 & 0 \\
\hline Other & 0 & 0 \\
\hline 15. What would you do if you thought you had symptoms of scabies? (Check all that apply.) & & \\
\hline Go to health facility & 292 & 67 \\
\hline Go to pharmacy & 48 & 11 \\
\hline Got to traditional healer & 81 & 19 \\
\hline Pursue other self-treatment options & 0 & 0 \\
\hline Other & 12 & 3 \\
\hline 16. Which statement is closest to you're feeling about people with scabies disease? & \\
\hline I feel compassion and desire to help & 118 & 27 \\
\hline Feel compassion but I tend to stay away from these people. & 207 & 48 \\
\hline It is their problem and I cannot get scabies. & 19 & 4 \\
\hline I fear them because they may infect me. & 61 & 14 \\
\hline I have no particular feeling. & 28 & 6 \\
\hline Other (please explain): & 0 & 0 \\
\hline 17. In your community, how is a person who has scabies usually regarded/treated & \\
\hline Most people reject him or her & 47 & 11 \\
\hline Most people are friendly, but they generally try to avoid him or her & 319 & 74 \\
\hline The community mostly supports and helps him or her & 67 & 15 \\
\hline Other (please explain): & 0 & 0 \\
\hline
\end{tabular}

\section{Discussion:-}

More than half of the students are not aware about scabies (56\%) and the students are representing community they belong to. Therefore, we could say that community awareness on scabies is poor. Majority of the students are young adults aged lees than 20 years and the school system does not allow educating them on the personal hygiene and diseases prevention. Similarly we may have such situation in the rural areas and remote villages that level of education on the disease preventions is very low. Luckily $67 \%$ of the students are willing to go the clinic for health seeking which is a good signed and we need to further strengthen the level of education among the community for early seeking help from the medical facilities. Seeking support for the treatment of Scabies cases from the health workers and health facility can be defined as opportunity that we can build and better support of public health service providers by the community.

About $44 \%$ of the respondent reported that sharing cloths and blanket can spread the scabies. The health educators could build on these facts to adopt their educational tools to the level of understanding of audience. Respondents provided mixed types of response in connection with whom they will see for their treatment and this indicates that scabies treatment will not results into total cure and people have received partial treatments ( poor control ). Health education on scabies treatment must be strengthened to give clear un ambiguous messages to people for scabies treatment.

People show a good degree of sympathy and compassion to support patients with scabies. Public are compassionate about scabies patients and are willing to support and this fact help the mangers and planners to focus on community support and awareness in scabies control.

Looks like that radio, family, friends, neighbors and colleagues are good sources for getting the information about scabies. This situation is indicating that either public health managers did not use the printed materials for scabies awareness or there is certain level of local cultures affected that public health awareness on scabies only remind focus with radio broadcasting.

Religious school does not have planned to support the principle of school for better plan on control of scabies. Advocacy for resources generation to support religious school. Provincial health officers must be mobilized to support the religious school for the screening and controlling of scabies. 


\section{References:-}

1. Hotez PJ, Molyneux DH, Fenwick A, Kumar Esan J, Ehrlich Sachs S, et al. (2007) Control of neglected tropical diseases. N Engl J Med 357: 1018-1027.

2. Feldmeier H, Heukelbach J (2009) epidermal parasitic skin diseases: a neglected Category of povertyassociated plagues. Bulletin of the World Health Organization 87: 152-159.

3. Heukelbach J, Feldmeier H (2006) Scabies. The Lancet. 367: 1767-1774.

4. World Health Organization (Accessed November 9, 2015)

5. Romani L, Steer AC, WhitfeldMJ, Kaldor, JM (2015) Prevalence of scabies and impetigo worldwide: a systematic review. The Lancet 5: 960-967.

6. UN report on control of communicable diseases -Asia: Copyright (c) UN Office for the Coordination of Humanitarian Affairs 2004

7. American Academy of Dermatology website. Available at: http://www.aad.org/ . Accessed July 14, 2009.

8. Berkow R, Beers MH, Fletcher AJ, Bogin RM. The Merck Manual of Medical Information-Home Edition. New York, NY: Simon and Schuster, Inc; 2000.

9. Centers for Disease Control and Prevention. Sexually Transmitted Diseases Treatment Guidelines, 2010. MMWR 2010; 59(No. RR-12):1-110.

10. Currie BJ, McCarthy, JS. Permethrin and ivermectin for scabies. New Engl J of Med. 2010: 362:717-725.

11. Hu S, Bigby M. Treating scabies: results from an updated Cochrane review. Arch Dermatol. 2008:144:163840.

12. Leone PA. Scabies and pediculosis pubis: an update of treatment regimens and general review. Clin Infect Dis. 2007 Apr 1; 44 Suppl 3:S153-9. Review. Available at: http://cid.oxfordjournals.org/content/44/Supplement\%5F3/S153.long.

13. Lindane shampoo and lindane lotion. Center for Drug Evaluation and Research. US Food and Drug Administration website. Available at: http://www.fda.gov/Drugs/DrugSafety/PostmarketDrugSafetyInformationforPatientsandProviders/ucm1 10452. htm. Accessed July

14. A Guide to Developing Knowledge Attitude and Practice Surveys

15. Provincial Health Management Information reports of the year 2016 